\title{
NEUROCIENCIA APLICADA AL CINE: MEDICIÓN DE LA ATENCIÓN Y LA EMOCIÓN EN LA AUDIENCIA DE “MEMORIA DE UN CINE DE PROVINCIAS"
}

NEUROCIÊNCIA APLICADA AO FILMES: MEDIÇÃO DA ATENÇÃO E EMOÇÃO NA AUDIÇÃO "MEMORIAS DE UN CINE DE PROVINCIAS" NEUROSCIENCE APPLIED TO THE CINEMA: MEASURING ATTENTION AND EMOTION PARAMETERS IN THE AUDIENCE OF THE FILM "MEMORIAS DE UN CINE DE PROVINCIAS" (MEMOIRE OF A PROVINCIAL CINEMA)

Alejandro Tapia Frade (Alejandro.tapia.frade@gmail.com)*

Elena Martín Guerra (emartin@sociograph.es) ${ }^{\star \star}$

\section{RESUMEN}

En este trabajo se muestran los resultados de un experimento de neurociencia sobre el cortometraje Memorias de un Cine de Provincias, donde se midió la actividad electrodérmica de un grupo de 32 personas de forma simultánea mediante la tecnología Sociograph.

Los resultados muestran una curva sostenida de atención durante toda la proyección, en una potencia situación de atención por expectación. Los picos emocionales más intensos se refieren a momentos previos a un desenlace, esperado o no. Dicha emoción fue verbalizada en términos positivos, de alegría y felicidad en la encuesta aplicada al término de la emisión.

Palabras-llave: Neurociencia, cine, atención, emoción, audiencia / Neuroscience, film, attention, emotion, audience.

\section{RESUMO}

Neste trabalho, mostram-se os resultados de uma experiência em neurociência sobre a curta metragem "Memorias de un cine de provincias", na qual a atividade electro dérmica foi medida simultaneamente num grupo de 32 pessoas pela tecnologia Sociografica. Os resultados mostram uma acentuada curva de atenção ao longo da projeção, numa situação de atenção por expectativa. O mais intenso dos picos emocionais foi registado nos momentos prévios a um desenlace, esperado ou não. Essa emoção foi verbalizada em termos positivos de alegria e felicidade na pesquisa realizada no final da transmissão.

Palavras-clave: Neurociência, cinema, atenção, emoção, audição.

\begin{abstract}
This article demonstrates the results of an experiment in neuroscience on the short film, "Memorias de un cine de provincias" ["Memoire of a Provincial Cinema], in which the electrodermic activity of a group of 32 people was measured simultaneously using Sociograph techniology. The results show a progressive curve in attention throughout the entire session, potentially related to their expectations. The most intensive emotional peaks were related to moments just proceeding the expected event (or not). This emotion was
\end{abstract}




\section{Eqitania \\ $s$ c i e $\Omega$ i a}

verbalized in positive terms, in joy and happiness on the questionnaire that was completed at the end of the film.

Keywords: Neuroscience, film, attention, emotion, audience.

*Licenciado en Publicidad y Relaciones Públicas (Univ. Complutense de Madrid) y Doctor en Marketing (Univ. Complutense de Madrid). Autor de una cuarentena de artículos en revistas indizadas y capítulos de libro. Acreditado ANECA y ACSUCYL a la figura de Profesor Contratado Doctor, con un sexenio de investigación (ACSUCYL). Profesor Agregado en la Universidad Europea Miguel de Cervantes, Valladolid, España.

** CEO y COFounder Sociograph Neuromarketing.Licenciada en Publicidad y Relaciones Públicas (Univ. Europea Miguel de Cervantes) y en Periodismo (Univ. de Valladolid). Doctoranda Univ. Complutense de Madrid.

Submitted: 12th May 2015

Accepted: 05th November 2015 


\section{INTRODUCCIÓN}

En la actualidad vivimos un contexto de saturación audiovisual donde sólo los productos más potentes, más impactantes, consiguen ser rentables. Por ello, y asumiendo que el coste de producción audiovisual (y por ello el riesgo que se asume) es en términos generales alto, resulta interesante la investigación de la audiencia de la forma más precisa posible.

Sin embargo, la psique de la audiencia es un fenómeno de extraordinaria complejidad. Las encuestas de opinión a menudo no son capaces de responder a las cuestiones requeridas con la debida precisión, ya que la mayor parte de los procesos psíquicos son internos, por tanto no observables, y no siempre su verbalización es la adecuada, frecuentemente porque los individuos encuestados ni siquiera son conscientes de ellos.

Por tanto, resulta en todo punto interesante el estudio de tipo psicobiológico de la audiencia. En la actualidad los métodos más usados son la tomografía por emisión de positrones, la imagen por resonancia magnética funcional, el electroencefalograma, el magnetoencefalografía y la actividad electrodérmica (Touhami et al, 2011:1528), La importancia de estos estudios es creciente desde hace unos años, a pesar de su novedad -el término neuromarketing emerge en 2002- (Morin,2011:131, Blakeslee, S.:2004)

La denominada actividad electrodérmica, es, por su sensibilidad, fiabilidad y facilidad de medición, uno de los medios más usados para medir atención y emoción, por lo se utiliza en este trabajo, pero con una peculiaridad: se mide de forma simultánea a un grupo de personas mediante una tecnología llamada Sociograph, cuya viabilidad ya ha sido testada anteriormente por Martínez Herrador (2012). La actividad electrodérmica genéricamente considerada ha sido también usada entre otros por Oxley (2008) y Garzón (2008).

Es, en suma, un fenómeno psicobiológico descubierto por Feré en 1881. Los mecanismos psicobiológicos implican modificaciones de las propiedades bioeléctricas de la piel por modificaciones de sus estructuras plasmáticas y en la actividad de las glándulas sudoríparas, inervada por el sistema nervioso simpático, responsable de nuestros procesos de activación . (Martínez Herrador, J.L. , Monge Benito, S. y Valdunquillo Carlón, M.I., 2012: 54)

La actividad electrodérmica (EDA) es muy usada en investigaciones de ciencias sociales, por su alta capacidad para responder a estímulos nuevos, afectivos, amenazantes y también cognitivos, así como a situaciones de ansiedad y estrés (Beer, 2007: 69-86). Se puede clasificar en tres categorías:

1)En primer lugar, la actividad tónica -EDL— (electrodermal level) que nos indicaría niveles basales de activación con una importante implicación en los procesos de atención. Los valores elevados de EDL indicarían mayores niveles de activación-atención, por tanto, más predisposición a recibir, analizar y responder a la información.

2) En segundo lugar, la actividad fásica - EDR - (electrodermal response) son respuestas psicofisiológicas especificas que se refieren a los rápidos cambios en la conductividad 


\section{Eqitania}

$s$ c i e $\Omega$ c i a

producidos por estímulos desencadenantes desconocidos o conocidos y controlados, en su caso, por el investigador. Su fundamento se basa en el reflejo de orientación. Es un buen predictor de cambio tras la exposición a un estímulo y es, por tanto, uno de los índices más utilizados.

3) En tercer lugar, la actividad espontánea — NSA — (non specific activity) se refiere a aquella actividad psicofisiológica no específica que no es posible atribuir a un desencadenante conocido, al no estar controlada su presentación. Son respuestas situacionales que representan el índice o grado de activación del sujeto, de manera que a mayor NSA, mayor actividad espontánea presenta el sujeto. Puede considerarse como "ruido" en los registros (Nikula, 2007:86-90; Martínez Herrador, J.L., Monge Benito, S. y Valdunquillo Carlón, M.I., 2012: 56).

En este artículo mostramos los resultados de un experimento de neurociencia, en el que analizamos mediante actividad electrodérmica los niveles de atención y emoción generados lo largo de la proyección del corto Memorias de un Cine de Provincias. En ella, el cineasta, pintor y escritor, Ramón Margareto, quiso reflejar la autobiografía de los cines Ortega de Palencia con motivo de su 60 aniversario, el cual fue también el lugar que acogió la proyección experimental de este cortometraje para evaluar el nivel de atención y emoción que provoca en la audiencia, y cuyos resultados aquí se presentan.

El corto Memorias de un Cine de Provincias resulta especialmente apto para la medición experimental que hemos propuesto debido a su carácter integrador respecto del cine convencional. Este corto, consta en sí de secuencias de afamadas obras como Blancanieves, candilejas, siete Novias para siete hermanos, el último cuplé, el puente sobre el río Kwai, Ben Hur, Mary Poppins, dos hombres y un destino, el espíritu de la colmena, terremoto, enmanuelle, la fuerza del cariño, Thelma y Louise, Ghost e Instinto Básico.

Además, ha obtenido otros premios en los festivales de cine de Zaragoza y Alicante, también en 2011, y fue galardonado como MON-DOC. Mostra Internacional de Cinema Documental de Montaverner (Valencia). el Mejor Relato Cinematográfico del Festival de Cine de Astorga. También ha sido proyectado en la gala inaugural de MON-DOC, Mostra Internacional de Cinema Documental de Montaverner (Valencia), y el Festival de Cine de Aguilar de Campoo, y en gala de clausura del Festival Internacional de Cortometrajes (FIC) de Móstoles.

Ramón Margareto destaca por sus aportaciones al cine y a la pintura. Ha dirigido la Muestra de Cine Internacional de Palencia, la Semana de Cine Español en Carabanchel y el Festival de cine de Aguilar de Campoo. Además, ha formado parte del jurado en una veintena de certámenes, es miembro del comité asesor del Festival de cine de Alicante y vicepresidente del Fórum Intercultural. Después de participar en numerosas muestras colectivas, en 2010 arrancó su carrera como pintor, con varias exposiciones individuales. Su filmografía está compuesta por un largometraje documental (Salamandras y salamandros), dos mediometrajes documentales (La silla eléctrica y Festiva), cinco cortometrajes de ficción (Amor digital, Supermoco, Viaje interior, La amenaza del coloso y Las primeras veces, adaptación de la novela de Jordi Mollá). Además, Ramón Margareto ha realizado cuatro anuncios publicitarios. 


\section{METODOLOGÍA}

La muestra realizada está conformada por 32 personas de ambos sexos y diferentes estratos de edad. Además, se trata de una muestra diseñada a conveniencia de los investigadores, siendo por tanto de carácter exploratorio al no tener carácter representativo alguno.

La distribución por sexo y edad se muestra en la tabla siguiente:

\begin{tabular}{|l|c|}
\hline Sexo & $\mathrm{n}$ \\
\hline Mujeres & 12 \\
\hline Hombres & 20 \\
\hline Total & 32 \\
\hline Edad & $\mathrm{n}$ \\
\hline De 14 a 24 años & 12 \\
\hline De 25 a 34 & 5 \\
\hline De 35 a 44 & 5 \\
\hline De 45 a 54 & 5 \\
\hline De 55 y más años & 32 \\
\hline Total & \\
\hline
\end{tabular}

Tabla I- Distribución de la muestra

Para el desarrollo de esta investigación se diseñó una metodología mixta que contemplaba, por un lado, la medición objetiva de las emociones y el grado de atención, y, por otro, la opinión respecto al cortometraje, utilizando un cuestionario semiestructurado.

Para obtener la medición objetiva del grado de atención y las reacciones emocionales durante la emisión del corto "Memorias de un cine de provincias", se utilizó el instrumento de medición Sociograph (ya usado en casos anteriores como Martínez Herrador, J.L. et al (2008), y Aiger, M. et al (2013)), patente n 9902767, que mide la actividad eletrodérmica en los dedos índice y medio de la mano izquierda usando un modelo de series temporales.

Se realizaron dos pases de 16 personas el día 22 de julio de 2013 en el cine Ortega de la ciudad de Palencia. A la entrada del recinto de emisión se colocaba a los participantes un brazalete con el instrumento de medición. Durante las proyecciones, el brazalete enviaba la información de cada individuo a una sistema central de proceso, con objeto de analizar en tiempo real los niveles de atención y emoción de los participantes para su posterior análisis.

El instrumento de medición analiza la actividad electrodérmica enviando a la unidad central de proceso tres tipos de señales:

1)La actividad tónica, relacionada con la atención (EDL). La unidad de medida usada es el sumatorio de la resistencia electrodérmica en Kilomnios $(K \Omega)$ de todos los participantes. Es importante señalar que a menor resistencia, mayor nivel de atención. 


\section{Egitania}

s c i e $\cap$ c i a

2) La actividad fásica, relacionada con la emoción (EDR). En este caso, la unidad de medida es la media aritmética de la resistencia electrodérmica en Kilomnios ( $K \Omega$ ) de todos los participantes. En este caso, a mayor media, mayor intensidad de emoción. Es de destacar que la máquina detecta simplemente la presencia de emoción, pero no el tipo o contenido de ésta, no discriminando por tanto entre emociones positivas y negativas.

3) Una señal espontánea, no específica, aleatoria e independiente de cada persona (NSA). La unidad de medida usada es la resistencia electrodérmica en Kilomnios (K $\Omega$ ). Esta señal, por ser inespecifica y subjetiva, propia de cada persona, se compensó mediante la media aritmética global para posteriormente poder discriminarla y despreciarla.

La herramienta de neuromarketing Sociograph mide estos parámetros con una frecuencia de 36 inputs por segundo. No obstante, para simplificar tanto la información como el proceso del análisis, se aporta un solo dato por segundo, resultante de centralizar a través del promedio aritmético esos 36 datos por segundo.

Posteriormente, tras la emisión del corto, se pedía la cumplimentación del cuestionario, de tipo auto administrado.

\section{RESULTADOS}

\subsection{NIVEL DE ATENCIÓN}

El nivel de atención que logra Memorias de un cine de provincias podemos considerarlo elevado, con un valor medio total de 225,24 Kiloohmios (K $\Omega$ ). Cabe mencionar, antes de presentar con detalle los resultados, que el nivel de atención se mide en función de la resistencia, de manera que a menor resistencia, mayor grado de atención, y viceversa.

El cortometraje logra mantener la atención de la audiencia en unos niveles estables durante la gran parte de la duración del mismo, si bien es cierto que es en los primeros minutos cuando se logra el mayor grado de atención. En el gráfico 1 se refleja la curva de atención resultante de toda la proyección y se pueden observar claramente estos niveles.

Es en el inicio de la proyección, como decimos, cuando se obtienen parámetros más altos en lo que a la atención se refiere. De hecho, en los primeros segundos se registran los valores más altos, con una resistencia mínima en torno a los $150 \mathrm{~K} \Omega$. A partir de este punto, el grado de atención se relaja de modo lineal, con una evolución progresiva hasta el momento en que la voz en off termina la presentación del cine Ortega, alcanzando para entonces valores en torno a los $210 \mathrm{~K} \Omega$ de nuestra escala (a los 2 minutos y 54 segundos de la proyección).

Posteriormente, el cortometraje consigue mantener la atención de la audiencia en unos niveles estables y constantes aunque, lógicamente, ya no con la misma intensidad del tramo inicial. El grado de atención se mantiene, aunque alcanza su punto más bajo al inicio del minuto 13 de la proyección, momento que viene a coincidir con las secuencias de la película Carros de Fuego, alcanzándose entonces el nivel de atención más moderado de todo el corto (249,98 K $\Omega$ ). Sin 
duda alguna, en este momento se detecta el mayor grado de relajación de la audiencia, con una duración aproximada de 20, superiores en más de un 10\% a la media de atención obtenida para todo el corto.

No obstante, a partir de este momento, el cortometraje logra incrementar de nuevo el nivel de atención hasta el final, consiguiendo mantener un rango mantenido con valores en torno a los $240 \mathrm{~K} \Omega$, e incrementándose en los últimos segundos, con las imágenes, nuevamente, del cine Ortega.

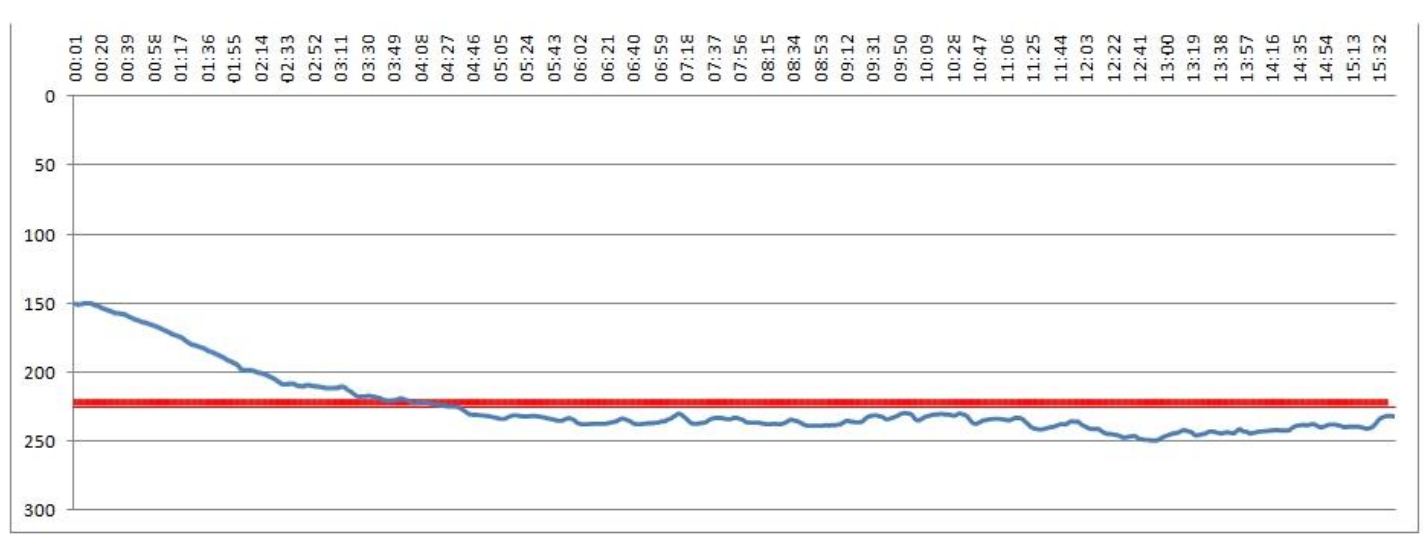

Figura 1-Curva EDL de atención en Memorias de un Cine de Provincias

Todo lo comentado anteriormente se ve ratificado cuando observamos los datos de la gráfica por tramos de la proyección. Se comprueba cómo el mayor grado de atención se registra, especialmente, en el primer tramo del corto, tal y como reflejan los resultados de la siguiente tabla, que reflejan menor resistencia en esos momentos. (recordamos que, a menor resistencia, mayor grado de atención).

\begin{tabular}{|l|c|}
\hline Tramo de tiempo (minutos:segundos) & $K \Omega$ \\
\hline Hasta 05:00 & 197,2826 \\
\hline Del 05:01 a 10:00 & 235,5633 \\
\hline Del 10:01 hasta el final & 239,7297 \\
\hline Intensidad media Global EDL & 225,2385 \\
\hline
\end{tabular}

Tabla II- Intensidad media de atención (EDL) en $K \Omega$

Esta curva de atención, con una evolución relativamente sostenida a lo largo de toda la proyección, podría deberse a la expectación que provoca en los espectadores la curiosidad del "qué vendrá ahora" ya que el recorrido de ejemplos cinematográficos tiende a la actualidad, por lo que esa expectación y esa atención se consiguen mantener para descubrir cuáles serán esas películas. 


\subsection{INTENSIDAD DE LAS REACCIONES EMOCIONALES}

La intensidad media de las reacciones emocionales que provoca el cortometraje en la audiencia es de 1,1985 kilohmios; teniendo en cuenta que se registran valores dentro de un rango que va desde el 0 hasta el valor más elevado que es de 18,0908 K $\Omega$.

El siguiente gráfico refleja la curva de las emociones registradas a lo largo de la proyección.

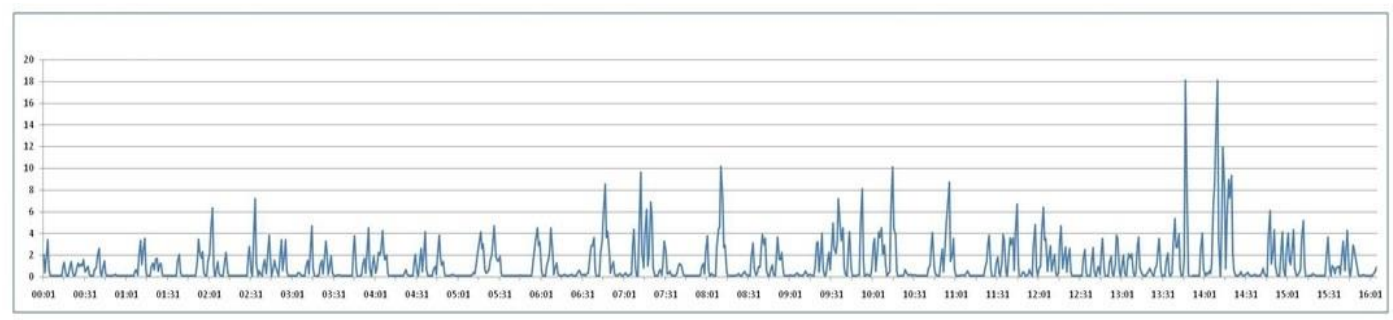

Figura 2- Curva EDR de emoción en Memorias de un Cine de Provincias

Analizando la evolución de este aspecto, se comprueba cómo el mayor grado de emoción se registra, especialmente, en el último tramo del corto, tal y como reflejan los resultados de la siguiente tabla.

\begin{tabular}{|l|c|}
\hline Tramo de tiempo (minutos:segundos) & $\mathrm{K} \Omega$ \\
\hline Hasta 05:00 & 0,8251 \\
\hline Del 05:01 a 10:00 & 1,2169 \\
\hline Del 10:01 hasta el final & 1,4901 \\
\hline Intensidad media Global EDR & 1,1985 \\
\hline
\end{tabular}

Tabla III- Intensidad media de emoción (EDR) en $K \Omega$

Sin embargo, y más allá de la evolución de las emociones en el transcurso del cortometraje, se detectan algunos momentos concretos de mayor intensidad claramente definidos.

Momentos concretos en los que se registra mayor intensidad en las reacciones emocionales (EDR) (en este caso, a mayor EDR mayor intensidad emocional).

\begin{tabular}{|c|l|r|}
\hline Momento (minuto:segundo) & Secuencia (película) & $\begin{array}{c}\text { EDR media } \\
\text { K } \Omega\end{array}$ \\
\hline $2: 03$ & Blancanieves & 6,3466 \\
\hline $2: 34$ & Escena esclavos & 7,2 \\
\hline $6: 47$ & Candilejas & 8,5183 \\
\hline $7: 13$ & Siete novias para siete hermanos & 9,6074 \\
\hline $7: 17$ & & 6,2043 \\
\hline
\end{tabular}




\begin{tabular}{|c|c|c|}
\hline $7: 20$ & El último cuplé & 6,8964 \\
\hline $8: 11$ & El puente sobre el río Kwai & 10,1658 \\
\hline $8: 12$ & Ben Hur & 7,3231 \\
\hline $9: 36$ & Mary Poppins & 7,166 \\
\hline $9: 53$ & Mary Poppins & 8,1192 \\
\hline $10: 14$ & Dos hombres y un destino & 6,1937 \\
\hline $10: 56$ & El espíritu de la colmena & 8,7137 \\
\hline $11: 45$ & Terremoto & 6,6926 \\
\hline $12: 04$ & Emmanuelle & 6,3678 \\
\hline $13: 47$ & La fuerza del cariño & 18,0908 \\
\hline $14: 10$ & Thelma y Louise & 18,0717 \\
\hline $14: 14$ & Thelma y Louise & 11,9575 \\
\hline $14: 20$ & Ghost & 9,3421 \\
\hline $14: 48$ & Instinto básico & 6,0918 \\
\hline & Intensidad media global EDR & 1,1985 \\
\hline
\end{tabular}

Tabla IV- EDR media $(K \Omega)$ por secuencia

Los momentos que más movilizan emocionalmente corresponden con La fuerza del cariño $(18,09 \mathrm{~K} \Omega)$ y las secuencia de Thelma y Louise $(18,07 \mathrm{~K} \Omega)$.

Resulta interesante comprobar cómo se dan altos niveles de coincidencia entre una mayor actividad emocional por parte de la audiencia, con la proyección de momentos previos a un desenlace esperado, incluso aunque este desenlace se conozca de antemano. De hecho, en el momento del desenlace, la intensidad emocional se relaja bastante con respecto a esos momentos previos.

Así, el grado de intensidad emocional es superior al detectado a lo largo de toda la proyección en los momentos previos al beso entre los personajes que encarnan Demi Moore y Patrick Swayze, en la película Ghost; o en el momento previo al famoso cruce de piernas de Sharon Stone en Instinto básico; y, sobre todo, la intensidad emocional que logra mantener durante un buen tiempo Ridley Scott en la escena de la persecución a Thelma y Louise, antes de decidir precipitarse por el barranco.

Además también, estos fragmentos, coinciden con el discurso de la voz en off cuando narra sus experiencias más emotivas y explica "lloré cuando".

Observamos, por tanto, como a través de Memorias de un cine de provincias, y de una medición objetiva, el cerebro humano se activa emocionalmente, mucho más en los momentos previos a un desenlace, que, en el momento del desenlace en sí, constituyendo por tanto un acto de emoción por expectación. 


\subsection{OPINIÓN}

Se analiza a continuación la opinión de la audiencia a partir de los resultados obtenidos de las respuestas al cuestionario de consulta respecto al cortometraje.

Las dos primeras cuestiones se refieren a los niveles de agrado e interés del cortometraje en la audiencia, formuladas del siguiente modo:

1) En su conjunto, ¿en qué grado te ha gustado el cortometraje? Posiciónate en una escala de 1 a 10, en la que el 1 es igual a "no me ha gustado nada" y el 10, "me ha gustado mucho".

2) En su conjunto, ¿en qué grado te ha resultado interesante? Posiciónate en una escala de 1 a 10, en la que el 1 es igual a "no me ha interesado nada" y el 10, "me ha resultado muy interesante".

Y, en general, la audiencia muestra una opinión favorable al corto, como demuestran los resultados de la siguiente tabla, en los que se comprueba cómo la mayor parte de los consultados se posicionan en los valores 7 y 8 de la escala, para unas valoraciones medias superiores al 6,00.

\begin{tabular}{|l|c|c|}
\hline \multicolumn{1}{|c|}{ Valoración } & Nivel de agrado & Nivel de interés \\
\hline Hasta 4 & 15,6 & 18,8 \\
\hline 5 y 6 & 25 & 21,9 \\
\hline 7 y 8 & 46,9 & 43,8 \\
\hline 9 y 10 & 12,5 & 15,6 \\
\hline Total & 100 & 100 \\
\hline Valor medio & 6,38 & 6,47 \\
\hline
\end{tabular}

Tabla V. Distribución de la audiencia por los diferentes niveles de agrado e interés en el cortometraje, en porcentaje

Continuando con el análisis del agrado, pedimos a los participantes en la investigación que indicaran, de modo abierto, aquello que más y menos les hubiera gustado de la proyección.

La vinculación del cortometraje con la ciudad parece un aspecto de importancia para la audiencia, de manera que son bastantes los que lo mencionan como el aspecto que más les ha gustado, mientras que otros lo consideran como el que menos, al considerar que el corto no aporta una vinculación suficiente a la ciudad de Palencia.

También, entre los aspectos más valorados está el repaso del corto a la historia del cine y, más concretamente, a películas específicas. Por el contrario, los aspectos técnicos (en especial, aquellos relacionados con la voz en off) son lo que menos han gustado a la audiencia participante en esta investigación. 


\begin{tabular}{|l|c|c|}
\hline Aspectos que más han gustado & $\%$ & (n) \\
\hline La mención a una película concreta & 25 & 8 \\
\hline La vinculación del corto con la ciudad & 21,8 & 7 \\
\hline El repaso a la historia del cine & 25 & 8 \\
\hline Algunas bandas sonoras concretas & 9,4 & 3 \\
\hline Otros & 9,4 & 3 \\
\hline No contestan & 9,4 & 3 \\
\hline Total & 100 & 32 \\
\hline Aspectos que menos han gustado & $\%$ & $(\mathrm{n})$ \\
\hline La mención a una película concreta & 9,4 & 3 \\
\hline Algunos aspectos técnicos & 37,5 & 12 \\
\hline La escasa vinculación del corto con la ciudad & 18,7 & 6 \\
\hline Otros & 9,4 & 3 \\
\hline No contestan & 25 & 8 \\
\hline Total & 100 & 32 \\
\hline
\end{tabular}

Tabla VI- Distribución de la audiencia (en porcentaje y $n$ absoluto) por los aspectos que más y menos les han gustado del cortometraje

Por último, se analizan los resultados referentes a la atención y a las sensaciones que ha despertado el corto en esta audiencia. Comenzando por este último aspecto, la mayoría de los entrevistados dicen que el corto les ha despertado alegría y muy pocos dicen que les ha generado disgusto; las sensaciones de tristeza y felicidad son minoritarias, aunque son significativas las cantidades de los que aseguran haber sentido ambas sensaciones.

Así, el 6,3\% de la audiencia dice haber sentido disgusto, el 37,5\% manifestó haber sentido tristeza, el 46,9\% que dicen haber sentido felicidad y el 78,1\% señala haber sentido alegría durante la proyección.

Generalmente, y siempre según la opinión de los entrevistados, los que aseguran haber sentido tristeza, la asocian a imágenes calificadas como tristes mencionando, especialmente, la película de Sydney Pollack, Memorias de África.

Por su parte, los que dicen haber sentido felicidad en algún momento de la visualización, asocian esta sensación a las películas más antiguas, que tienen que ver con recuerdos de la juventud o de lugares de la ciudad.

Por último, la gran mayoría de los que aseguran haber sentido alegría, lo asocian a una amplia diversidad de las películas mencionadas a lo largo del cortometraje, mientras que no se obtiene ninguna asociación concreta de los pocos que dicen haber sentido disgusto.

En cuanto al momento que más atención ha provocado, los encuestados podían verbalizar aquellos dos que más les hubiera llamado la atención. Los 32 entrevistados aportan 57 
menciones diferentes, con mayor incidencia en las escenas en que se menciona alguna relación con la ciudad y algunas películas concretas, entre las que destacan, por este orden, las menciones a Parque Jurásico, ET, el extraterrestre, Carros de Fuego y Memorias de África.

La siguiente tabla refleja la distribución de los entrevistados según estas menciones sobre la atención.

\begin{tabular}{|l|c|}
\hline Momento & $\mathrm{n}$ \\
\hline Momentos con vinculación a la ciudad & 7 \\
\hline Parque jurásico & 6 \\
\hline ET, el extraterrestre & 4 \\
\hline Carros de fuego & 4 \\
\hline Memorias de África & 2 \\
\hline Titanic & 2 \\
\hline Terminator 2 & 2 \\
\hline El Rey León & 2 \\
\hline Lo que el viento se llevó & 2 \\
\hline Love story & 2 \\
\hline El último cuplé & 2 \\
\hline Cantando bajo la lluvia & 2 \\
\hline Instinto básico & 2 \\
\hline 200l, Odisea en el espacio & 2 \\
\hline Jesucristo superstar & 1 \\
\hline Casablanca & 1 \\
\hline El Mago de Oz & 1 \\
\hline Sonrisas y lágrimas & 57 \\
\hline Blancanieves & 1 \\
\hline La bella y la bestia & 1 \\
\hline Otros momentos sin especificar & 2 \\
\hline Momentos de bandas sonoras & 2 \\
\hline Total & 2 \\
\hline
\end{tabular}

Tabla VII- Distribución de los entrevistados, según los momentos que dicen que más les ha llamado la atención

\section{CONCLUSIONES}

Memorias de un cine de provincias obtiene de la audiencia un nivel de atención muy elevado al principio; posteriormente este grado de atención se relaja, manteniéndose en niveles similares a lo largo de toda la proyección.

Parece que este grado de atención logra incrementar su intensidad cuando aparecen contenidos relacionados con la ciudad de Palencia, especialmente con las imágenes del cine Ortega en una audiencia, recordemos, de la propia ciudad. 
De hecho, la audiencia muestra, de modo verbalizado, sus preferencias por la vinculación del cortometraje a la ciudad. Así, una de las mayores proporciones de consultados lo señalan como el aspecto que más les ha agradado, mientras que otra proporción importante considera que existe escasa vinculación y lo indican como el aspecto que menos les ha gustado. Entre ambas opiniones, tenemos al $40 \%$ de los consultados.

En cierto modo, se detecta cierta relación entre la atención objetiva medida en Kilomnios y la atención verbalizada, al ser la vinculación del corto con la ciudad el aspecto más relevante en uno y otro caso. No obstante, esta relación no parece muy intensa, ya que las respuestas verbalizadas de la audiencia sobre los momentos de mayor atención se dispersan bastante por diferentes momentos de la proyección.

Por otra parte, el cortometraje logra un amplio número de picos emocionales intensos; estos momentos coinciden con los previos al desenlace de una historia, tal y como sucede en las secuencias previas al desenlace definitivo de películas como Thelma y Louise o Ghost, por ejemplo.

Finalmente, en relación al contenido emocional, es interesante destacar que proporciones elevadas de audiencia verbalizan emociones positivas, relacionadas con la felicidad y, sobre todo, la alegría; en cambio, son minoría los que hablan de haber experimentado tristeza y, muy pocos, los que dicen haber sentido disgusto. No podemos establecer una relación directa entre las emociones experimentadas y las verbalizadas debido las restricciones metodológicas y técnicas de la propia investigación.

Al hilo de lo expuesto, resultaría interesante abordar la cuestión de la medición objetiva, no verbalizada, del contenido emocional, que podría ayudar a narrar con mayor precisión, o en su caso a explotar comercialmente a los personajes en forma de merchandising, publicidad, product placement u otras fórmulas de marketing.

\section{REFERENCIAS}

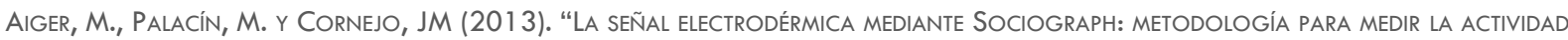
GRUPAL" REVISTA INTERNACIONAL DE PSICOLOGÍA SOCIAL: INTERNATIONAL JOURNAL OF SOCIAL PSICHOLOGY, N²8, VOL. 3 PP.333-347

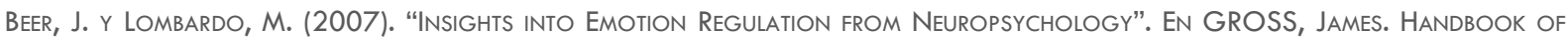
Emotion Regulation. NeW YORK: GuIlford PRESS, P. 69-86

Blakeslee, S. (2004). "If You Have a 'Buy Button' in Your Brain, What Pushes It?." New York Times, 19 de Octubre

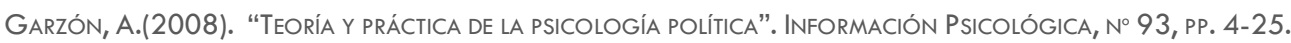

Martínez Herrador, Jl., MONGe Benito, S. y Valdunquillo Carlón, MI. (2012). "Medición de las ReSPuestas pSicofisiológicas Grupales PARA APOYAR EL ANÁLISIS DE DISCURSOS POLÍTICOS" TRIPODOS, N²9. PP. 53-72

Morín, C. (2011). "Neuromarketing: The New Science Of Consumer Behavior." Symposium: Consumer culture in Global perspective, $N^{\circ} 1$, PP. $131-135$

NIKULA, R. (2007). "PSYChOlogICAL CORRELATES OF NONSPECIFIC SKIN CONDUCTANCE RESPONSE.” PSYCHOPHYSIOLOGY. N²8, PP. 86-90. 


\section{Eqitania \\ s c i e $\Omega$ c i a}

Oxley, D. et al. (2008). "Political Attitudes Vary With Physiological Traits". Science, No321, pp. 1667-1670

Touhami, ZO. et Al. (2011). "Neuromarketing: Where marketing and neuroscience meet” African Journal Of Business Management № 5 , PP. 1528-1532 